\title{
Digestibilidad in vitro de dietas con diferentes niveles de inclusión de moringa (Moringa oleifera) para corderos en crecimiento
}

\author{
In vitro digestibility of diets with different levels of inclusion of moringa \\ (Moring olifera) for growing lambs
}

Jairo Jeú Quintanilla-Medina1, Daniel López-Aguirre', Juan Carlos MartínezGonzález, Andrés Gilberto Limas-Martínez', Froylán Andrés Lucero-Magaña1, Salomón Ruíz-García ${ }^{1,}$ Javier Hernández-Meléndez ${ }^{1,2}$

\section{Resumen}

\begin{abstract}
El presente estudio tuvo como objetivo evaluar diferentes niveles de inclusión de forraje de Moringa oleifera L en dietas para corderos en crecimiento sobre la cinética de fermentación ruminal y degradabilidad in vitro. Se utilizaron cinco tratamientos, los cuales fueron expresados en $\mathrm{g} \mathrm{kg}^{-1} \mathrm{MS}$ y consistieron en: control (IM0 = 0), nivel de inclusión bajo (IM10 = 100), niveles de inclusión medios (IM20 = 200 e IM30=300) y nivel de inclusión alto $(\mathrm{IM} 40=400)$. No se observó un efecto de los niveles de inclusión de moringa a las 6,12 y 24 h; sin embargo, a las 48 h el nivel de inclusión IM40 obtuvo el menor valor ( $\left.116.0 \mathrm{ml} \mathrm{g}^{-1}\right)$. En la producción de gas acumulada a las 48 y $72 \mathrm{~h}$ se observó un comportamiento lineal; es decir, a medida que aumenta el nivel de inclusión de $M$. oleifera en la dieta la producción de gas disminuye. Así mismo, se observó que la digestibilidad in vitro de la materia seca (DIVMS) fue afectada por el nivel de inclusión de M. oleifera, observándose una disminución de la degradabilidad conforme aumenta el nivel de inclusión, donde los niveles de 100 y $200 \mathrm{~g} \mathrm{~kg}^{-1} \mathrm{MS}$ igualaron al valor observado para la dieta testigo (796.3, 794.5 y $810.5 \mathrm{~g} \mathrm{~kg}^{-1} \mathrm{de} \mathrm{MS}$, respectivamente). Utilizar Moringa oleifera L a niveles menores al $20 \%$ en dietas para corderos en crecimiento representa una alternativa de alimentación para las unidades de producción.
\end{abstract}

Palabras clave: Moringa oleifera, rumiantes, digestibilidad, alimentación animal, producción de gas

\footnotetext{
${ }^{1}$ Facultad de Ingeniería y Ciencias, Universidad Autónoma de Tamaulipas, Centro Universitario Adolfo López Mateos, Ciudad Victoria, Tamaulipas, México

${ }^{2}$ E-mail: javhernan@docentes.uat.edu.mx
}

Recibido: 8 de octubre de 2019

Aceptado para publicación: 12 de junio de 2020

Publicado: 11 de agosto de 2020 
The study aimed to evaluate different levels of inclusion of Moringa oleifera L forage in diets for growing lambs on the kinetics of ruminal fermentation and in vitro degradability. Five treatments were used, expressed in $\mathrm{g} \mathrm{kg}^{-1} \mathrm{DM}$ and consisted of control $($ IM0 $=0)$, low inclusion level (IM10 = 100), medium inclusion levels (IM20 = 200 and IM30 = 300) and high inclusion level (IM40 = 400). No effect of moringa inclusion levels was observed at 6,12 and $24 \mathrm{~h}$; however, at $48 \mathrm{~h}$ the IM40 inclusion level obtained the lowest value $\left(116.0 \mathrm{ml} \mathrm{g}^{-1}\right)$. In the accumulated gas production at 48 and $72 \mathrm{~h}$, a linear pattern was observed, as the level of inclusion of $M$. oleifera in the diet increases, gas production decreases. Likewise, it was observed that the in vitro digestibility of dry matter was affected by the level of inclusion of $M$. oleifera, observing a decrease in degradability as the level of inclusion increases, where the levels of 100 and $200 \mathrm{~g} \mathrm{~kg}^{-1}$ MS equalled the value observed for the control diet $\left(796.3,794.5\right.$ and $810.5 \mathrm{~g} \mathrm{~kg}^{-1}$ of DM, respectively). Using Moringa oleifera L at levels less than $20 \%$ in diets for growing lambs represents a feeding alternative for production units.

Key words: Moringa oleifera, ruminants, digestibility, animal feed, gas production

\section{INTRODUCCIÓN}

Se dispone de diversos métodos para evaluar el valor nutricional de los alimentos suministrados al ganado, siendo uno de ellos la técnica de producción de gases (Posada y Noguera, 2005), la cual permite determinar la extensión y la cinética de degradación del alimento a través del volumen de gas producido durante el proceso fermentativo (Theodorou et al., 1994). Tiene la ventaja sobre otros métodos que puede cuantificarse el papel de los componentes solubles del sustrato durante el curso de la fermentación (Pell et al., 1997). Por otro lado Ceballos et al. (2008), mencionan que el sistema Daisy ${ }^{I I}$ (ANKOM Corp., Fairtport, NY, EEUU) se utiliza como método alternativo para calcular la degradación del alimento en el rumen en condiciones de laboratorio.

El forraje proveniente de árboles y arbustos puede utilizarse como alternativa en la suplementación del ganado en reemplazo total o parcial de concentrados, los cuales son de mayor costo y no contribuye a la degradación ambiental (Rodríguez, 2011).
La suplementación con forraje de Moringa oleifera Lam. (familia Moringaceae) es una eficiente vía para mejorar la utilización de dietas basales de baja a mediana calidad (Quintanilla-Medina et al., 2018). Astutia et al. (2011), mencionan que $M$. oleifera, siendo una planta tropical, tiene el mayor potencial para aumentar el suministro de nutrientes y mejorar la fermentación microbiana del rumen. En el estudio de Melesse (2001) con M. oleifera y $M$. stenopetala, reportaron valores para producción de gas in vitro de $47.9 \mathrm{ml}$ para $M$. stenopetala, siendo significativamente más alta que para $M$. oleifera $(40.6 \mathrm{ml})$. De manera similar, los valores de energía metabolizable - EM $\left(9.83 \mathrm{MJ} \mathrm{kg}^{-1}\right.$ de materia seca - MS), digestibilidad de la materia orgánica - DMO (76.4\%) y ácidos grasos de cadena corta - AGCC $(101 \mathrm{mmol})$ en $M$. stenopetala fueron significativamente más altos que los de $M$. oleifera.

Moringa oleifera y M. stenopetala son consideradas como fuentes de minerales, contienen un alto nivel de proteína cruda (PC), alta degradabilidad de la materia seca (MS) y de digestibilidad de materia orgánica 
(MO) (Debela y Tolera, 2013). El follaje de M. oleifera en tres edades de rebrote (45, 60 y 75 días) presenta una alta y fácil degradación potencial en el rumen, tanto de la MS con $92.5,98.8$ y $96.4 \%$, como de la MO con $66.8,73.5$ y $69.3 \%$, respectivamente. Por su parte, Rodríguez et al. (2014) en un estudio realizado con moringa muestran que la digestibilidad in vitro de la MS fue más del $50 \%$; asimismo muestran niveles de $\mathrm{MO}$ y fibra detergente neutra (FDN) de $59.34 \mathrm{y}$ $63.75 \%$, respectivamente. Por lo tanto, el objetivo del presente estudio fue evaluar varios niveles de inclusión de forraje de Moringa oleifera en dietas para corderos en crecimiento sobre la cinética de fermentación ruminal y degradabilidad in vitro.

\section{MATERIALES Y MéTOdOS}

\section{Localización del Estudio}

El estudio de producción de gas in vitro se realizó en el laboratorio de Nutrición Animal dentro de la Central Integral de Laboratorios de la Facultad de Ingeniería y Ciencias de la Universidad Autónoma de Tamaulipas, México.

\section{Hojas de Moringa oleifera}

Las muestras de $M$. oleifera fueron colectadas en una pradera de un año, establecida en la Posta Zootécnica «Ing. Herminio García González», ubicada en el municipio de Güémez, Estado de Tamaulipas, a 145 msnm, zona que predomina un clima semiseco y cálido (INEGI, 2019).

Las hojas se colectaron por la mañana y fueron secadas durante 72 horas bajo sombra y a temperatura ambiente $\left(35-40{ }^{\circ} \mathrm{C}\right)$, dentro de un invernadero colocando capas de $8-10 \mathrm{~cm}$ de espesor en mallas a $1.5 \mathrm{~m}$ de altura del suelo para mantener una mayor pérdida de humedad y evitar problemas de pudrición por hongos. Las hojas secas se tritu- raron utilizando un molino Thomas-Wiley Mill ${ }^{\circledR}$ (Thomas Scientific, USA) y tamizadas en una malla de $1 \mathrm{~mm}$ de diámetro de poro. Fueron almacenadas a una temperatura entre 22 y $35^{\circ} \mathrm{C}$.

\section{Tratamientos}

Se utilizaron cinco tratamientos, expresados en $\mathrm{g} \mathrm{kg}^{-1}$ MS: Testigo (IM- $0=0$ ); Nivel de inclusión bajo (IM-10=100); Niveles de inclusión medios (IM-20=200, IM-30=300); Nivel de inclusión alto (IM40=400). En el Cuadro 1 se presenta la composición química y nutrimental de las dietas experimentales.

\section{Obtención del Inóculo}

La obtención del líquido ruminal (inóculo) se realizó mediante la utilización de una sonda esofágica de $1 \mathrm{~cm}$ de diámetro y una bomba de vacío. Se extrajo líquido ruminal de cuatro corderos Pelibuey machos de peso $25 \pm 2.5 \mathrm{~kg}$ y 135 días de nacidos, alimentados en pastoreo continuo en praderas de pasto Bermuda variedades Tiftón 68 (Cynodon nlemfuensis Vanderyst) y 85 (Cynodon dactylon L. Pers.) establecidas bajo condiciones de riego. Los animales estuvieron en ayuno de $24 \mathrm{~h}$ con agua ad libitum. El líquido ruminal se trasladó al laboratorio en un recipiente térmico a $39-40{ }^{\circ} \mathrm{C}$. En el laboratorio fue filtrado en cuatro capas de gasa simple para retirar las partículas de alimento y se mantuvo bajo flujo constante de $\mathrm{CO}_{2}$ hasta la hora de inoculación.

\section{Inoculación e Incubación de las Mues- tras}

En los viales se depositaron $90 \mathrm{ml}$ de medio nutritivo, incluyendo los blancos y los estándares (maíz, soya y búffel) y $10 \mathrm{ml}$ de líquido ruminal. Se sellaron con un tapón de caucho y un anillo metálico a presión con una pinza, se agitaron se llevaron a incubación a $39^{\circ} \mathrm{C}$. 
Cuadro 1. Composición química y nutrimental de las dietas experimentales

\begin{tabular}{lccccc}
\hline \multirow{2}{*}{ Ingredientes } & \multicolumn{5}{c}{ Dietas experimentales $\left(\mathrm{g} \mathrm{kg}^{-1} \mathrm{MS}\right)$} \\
\cline { 2 - 6 } & $\mathrm{IM}-0$ & $\mathrm{IM}-10$ & $\mathrm{IM}-20$ & $\mathrm{IM}-30$ & $\mathrm{IM}-40$ \\
\hline Moringa oleifera & 0 & 100 & 200 & 300 & 400 \\
Sorgo & 330.9 & 319.0 & 307.2 & 295.3 & 283.5 \\
Soya & 180.9 & 153.5 & 104.5 & 65.6 & 26.5 \\
Búffel & 406.6 & 357,5 & 308.3 & 250.2 & 210.0 \\
Melaza & 50.0 & 50.0 & 50.0 & 50.0 & 50.0 \\
Minerales & 30.0 & 30.0 & 30.0 & 30.0 & 30.0 \\
Nutrientes & & & & & \\
$\quad$ Proteína cruda & 14.0 & 14.0 & 14.0 & 14.0 & 14.0 \\
$\quad$ Energía metabolizable & 2.70 & 2.70 & 2.70 & 2.70 & 2.70 \\
$\quad$ Mcal kg-1 MS) & & & & & \\
$\quad$ Fibra detergente neutro & 329.8 & 305.7 & 281.7 & 257.6 & 233.6 \\
$\quad$ Fibra detergente ácido & 172.1 & 161.2 & 150.4 & 139.5 & 128.6 \\
$\quad$ Materia orgánica & 901.9 & 899.7 & 897.5 & 895.3 & 893.1 \\
\hline
\end{tabular}

IM-0: dieta testigo; IM-10; IM-20, IM-30, IM-40: dietas con nivel de inclusión de Moringa oleífera de 10, 20, 30 y $40 \%$

Se utilizó un transductor de presión para medir la presión en psi (libras por pulgada cuadrada) de los gases acumulados en la parte superior de cada botella. Para cada medición, se perforaba los tapones de los viales con una aguja provista en el parte final del lector del transductor. Las mediciones se realizaron a las 2, 4, 6, 8, 10, 12, 15, 19, 24, 30, 36,48 y 72 h pos-incubación. Luego de cada lectura, las botellas se agitabas y se reubicaban en la estufa.

\section{Variables}

Volumen de gas producido ( $\left.\mathrm{ml} \mathrm{g}^{-1} \mathrm{MS}\right)$

La presión generada por el gas en la parte superior de los viales de incubación se midió a través de un transductor de presión conectado a un lector digital. La ecuación se obtuvo previamente usando PROC REG del programa SAS (año): $\mathrm{Y}=0.0854+(5.2869)$ $* \mathrm{X}$, donde: $\mathrm{Y}=$ es el volumen $(\mathrm{ml}), \mathrm{X}=$ es la presión (psi).

Los datos de producción de gas $\left(\mathrm{ml} \mathrm{g}^{-1}\right.$ MS) se ajustaron utilizando la opción NLIN de SAS (2002) al modelo de France et al. (2000) para el volumen de gas producido como $\mathrm{A}=b \times\left(1-e^{-c(t-L)}\right)$, donde $\mathrm{A}=$ es el volumen de producción de gas en el tiempo t; $b=$ es la producción de gas asintótico $(\mathrm{ml} \mathrm{g}$ $\left.\mathrm{MS}^{-1}\right) ; c=$ es la tasa de producción de gas $(/ \mathrm{h})$ de la fracción de alimento $b$ fermentable lentamente; $L=$ es el tiempo de retardo discreto antes de la producción de gas. 
Energía metabolizable (EM, $M J \mathrm{~kg}^{-1} \mathrm{MS}$ )

La energía metabolizable (EM, $\mathrm{MJ} \mathrm{kg}^{-1}$ MS) fue estimada de acuerdo con Menke y Steingass (1988) como sigue: EM (MJ kg MS $\left.{ }^{1}\right)=2.20+0.136$ PG24 (ml 0.2 $\left.\mathrm{g}^{-1} \mathrm{MS}\right)+\mathrm{PC}$, donde $\mathrm{EM}=$ energía metabolizable $\left(\mathrm{MJ} \mathrm{kg}^{-1}\right.$ MS); $\mathrm{PG} 24=$ volumen de gas producido a la hora $24(\mathrm{ml})$; y PC = proteína cruda $(\%)$ de cada muestra de Moringa oleifera.

Ácidos grasos de cadena corta (AGCC, mmol)

La concentración de ácidos grasos de cadena corta (AGCC, mmol) fue estimada utilizando la siguiente fórmula: $\mathrm{AGCC}=$ $-0.00425+0.0222$ (PG24 en ml), donde AGCC $=$ ácidos grasos de cadena corta (mmol); PG24 = volumen de gas producido a la hora $24(\mathrm{ml})$.

Digestibilidad in vitro de la materia seca (DIVMS \%) y materia orgánica (DIVMO \%)

La digestibilidad in vitro de la MS y MO se calculó mediante la recuperación de la fracción no degradada al final de la incubación. Cada uno de los viales fue filtrado al vacío a través de crisoles de vidrio (porosidad 1-100 a $160 \mu \mathrm{m}$ de tamaño de poro) secados a $100^{\circ} \mathrm{C}$ durante $24 \mathrm{~h}$, dejándose enfriar durante $5 \mathrm{~min}$ en un desecador y fueron pesados. Se calculó la DIVMS mediante la siguiente ecuación: DIVMS $(\%)=(\mathrm{W} 1-[\mathrm{WR}-\mathrm{WB}] / \mathrm{W} 1) * 100$, donde DIVMS $(\%)=$ digestibilidad in vitro de la materia seca en porcentaje; $\mathrm{W} 1$ = peso de la MS de la muestra en gramos; WR = peso de la MS del residuo en gramos; WB = peso de la MS del blanco en gramos.

Una vez registrado el peso de los crisoles se incineró la muestra a $500{ }^{\circ} \mathrm{C}$ por $6 \mathrm{~h}$ para realizar el cálculo de la DIVMO mediante la siguiente ecuación: DIVMO $(\%)=$ (W1MO - [WRMO - WBMO]/W1MO) * 100 , donde DIVMS $(\%)=$ digestibilidad in vitro de la MS en porcentaje; $\mathrm{W} 1 \mathrm{MO}=$ peso de la MO de la muestra en gramos; WRMO = peso de la MO del residuo en gramos;
WBMO $=$ peso de la MO del blanco en gramos.

\section{Análisis Estadístico}

En el caso de la producción de gas in vitro se ajustaron los datos obtenidos mediante el modelo de France et al. (2000). Los datos de la fermentación ruminal in vitro y la digestibilidad de los nutrientes fueron analizados mediante un diseño completamente al azar con cuatro tratamientos (IM-0, IM-10, IM-20, IM-30e IM-40) y 5 repeticiones, utilizando procedimiento general para modelos lineales (GLM) de SAS (2002). La prueba de Tukey se utilizó para separar las medias significativas $(\mathrm{p}=0.05)$. Se utilizó la prueba de contraste polinómico para determinar el efecto lineal y cuadrático de los niveles de inclusión de M. oleifera sobre todas las variables dependientes.

\section{Resultados}

\section{Producción de Gas in vitro}

No se observó efecto de los niveles de inclusión de $M$. oleifera en la producción de gas acumulada ( $\mathrm{ml} \mathrm{g}^{-1}$ de MS) de la fermentación in vitro de las dietas a las 6,12 y $24 \mathrm{~h}$. Sin embargo, a las 48 h se observó que el nivel de inclusión $\mathrm{IM}_{40}$ obtuvo el menor valor $\left(115.98 \mathrm{~mL} \mathrm{~g}^{-1}\right)$. En el caso de la producción de gas acumulada a las 48 y $72 \mathrm{~h}$, se observa un comportamiento lineal, donde al aumentar el nivel de inclusión de $M$. oleifera disminuye la producción de gas (Cuadro 2).

Los resultados de la cinética de producción de gas, valor energético, degradabilidad in vitro de la materia seca se presentan en el Cuadro 3. La asíntota de producción de gas (b) fue similar entre la dieta testigo (IM-0) y la dieta con el nivel de inclusión de $100 \mathrm{~g} \mathrm{~kg}^{-1}$ MS (IM-10), siendo estos superiores al resto de las dietas evaluadas. Se observó que a medida que incrementa el nivel de inclusión de $M$. oleifera en la dieta, la variable de pro- 
Cuadro 2. Producción de gas acumulada $\left(\mathrm{ml} \mathrm{g}^{-1}\right.$ de $\left.\mathrm{MS}\right)$ de la fermentación in vitro de dietas para corderos en crecimiento con diferentes niveles de Moringa oleifera

\begin{tabular}{lcccccc}
\hline \multirow{2}{*}{$\begin{array}{l}\text { Dietas } \\
\left(\mathrm{g} \mathrm{kg}^{-1} \mathrm{MS}\right)\end{array}$} & $\begin{array}{c}\text { Nivel de } \\
\text { inclusión } \\
\left(\mathrm{g} \mathrm{kg}^{-1} \mathrm{MS}\right)\end{array}$ & $6 \mathrm{~h}$ & $12 \mathrm{~h}$ & $24 \mathrm{~h}$ & $48 \mathrm{~h}$ & $72 \mathrm{~h}$ \\
\cline { 3 - 7 } & 0 & $9.49^{\mathrm{a}}$ & $32.73^{\mathrm{a}}$ & $72.53^{\mathrm{a}}$ & $131.12^{\mathrm{a}}$ & $169.76^{\mathrm{a}}$ \\
\hline Testigo & 100 & $9.34^{\mathrm{a}}$ & $31.31^{\mathrm{a}}$ & $68.75^{\mathrm{a}}$ & $123.26^{\mathrm{ab}}$ & $158.68^{\mathrm{ab}}$ \\
M. oleifera & 200 & $8.69^{\mathrm{a}}$ & $29.72^{\mathrm{a}}$ & $65.48^{\mathrm{a}}$ & $117.38^{\mathrm{ab}}$ & $150.98^{\mathrm{b}}$ \\
& 300 & $8.92^{\mathrm{a}}$ & $31.01^{\mathrm{a}}$ & $67.74^{\mathrm{a}}$ & $118.80^{\mathrm{ab}}$ & $149.93^{\mathrm{b}}$ \\
& 400 & $9.62^{\mathrm{a}}$ & $31.64^{\mathrm{a}}$ & $67.63^{\mathrm{a}}$ & $115.98^{\mathrm{b}}$ & $144.19^{\mathrm{b}}$ \\
& - & 1.064 & 2.120 & 3.276 & 3.894 & 0.0050 \\
Error estándar & - & 0.3295 & 0.4162 & 0.2558 & 0.0330 & 0.0032 \\
Valor-p & - & 0.3890 & 0.1493 & 0.0470 & 0.0044 & 0.0006 \\
Testigo vs & & & & & & \\
M. oleifera & - & 0.8857 & 0.4722 & 0.1274 & 0.044 & 0.0002 \\
$\quad$ Lineal & - & 0.0680 & 0.1000 & 0.1261 & 0.1771 & 0.2528 \\
\hline Cuadrático & - & & & & &
\end{tabular}

Valores dentro de columnas con diferente literal son estadísticamente significativos. Tukey $(p<0.05)$. EEM = Error estándar de la media

ducción de gas tiende a decrecer. Así mismo para la fracción $(c)$ se observó un comportamiento lineal donde a medida que incrementa el nivel de inclusión de $M$. oleifera, la velocidad a la que se produce el gas también se incrementa. Por otro lado, para la fase lag $(L)$ se observa que la dieta testigo y las dietas con niveles de inclusión de 100,200 y 300 $\mathrm{g} \mathrm{kg}^{-1} \mathrm{MS}$, iniciaron su producción de gas a la misma hora, y el nivel más elevado de inclusión de $M$. oleifera en la dieta obtuvo el menor valor $(3.60 \mathrm{~h})$ retardando el inicio de la producción de gas.

\section{Digestibilidad in vitro de la MS y MO}

La digestibilidad in vitro de la materia seca (DIVMS) y de la materia orgánica (DIVMO) fue afectada por el nivel de inclu- sión de M. oleifera, observándose una disminución de la degradabilidad conforme aumenta el nivel de inclusión, donde los niveles de 100 y $200 \mathrm{~g} \mathrm{~kg}^{-1}$ MS igualaron al valor observado para la dieta testigo (796.33, 794.52 y $810.49 \mathrm{~g} \mathrm{~kg}^{-1} \mathrm{de} \mathrm{MS}$, respectivamente). Así mismo, cabe mencionar que el valor más bajo de degradabilidad (dieta con $400 \mathrm{~g} \mathrm{~kg}^{-1}$ de MS) fue superior a $740 \mathrm{~g} \mathrm{~kg}^{-1} \mathrm{MS}$, lo cual es aceptable. Para las variables de EM y AGCC no se observó efecto del nivel de inclusión de M. oleifera.

En el Cuadro 4 se presentan los resultados del efecto de los niveles de $M$. sobre la digestibilidad in vitro de la materia seca y la fibra a las 24 y $48 \mathrm{~h}$ de incubación en las botellas de fermentación. Los resultados para DMS a la hora 24 muestran un comportamiento cuadrático, observándose también que 
Cuadro 3. Cinética de producción de gas, valor energético, degradabilidad in vitro de la materia seca de dietas para corderos en crecimiento con diferentes niveles de Moringa oleifera

\begin{tabular}{lccccccc}
\hline Dietas & $\begin{array}{c}\text { Nivel de } \\
\text { inclusión } \\
\left(\mathrm{g} \mathrm{kg}^{-1}\right. \\
\mathrm{MS})\end{array}$ & $\begin{array}{c}b \\
\left(\mathrm{~mL} \mathrm{~g}^{-1}\right)\end{array}$ & $\begin{array}{c}\mathrm{C} \\
(/ \mathrm{h})\end{array}$ & $\begin{array}{c}\mathrm{L} \\
(\mathrm{h})\end{array}$ & $\begin{array}{c}\text { DIVMS } \\
\left(\mathrm{g} \mathrm{kg}^{-1}\right. \\
\mathrm{MS})\end{array}$ & $\begin{array}{c}\mathrm{EM} \\
\left(\mathrm{MJ} \mathrm{kg}^{-1}\right)\end{array}$ & $\begin{array}{c}\text { AGCC } \\
(\mathrm{mmol})\end{array}$ \\
\hline Testigo & 0 & $244.85^{\mathrm{a}}$ & $0.0172^{\mathrm{b}}$ & $3.7165^{\mathrm{ab}}$ & $810.49^{\mathrm{a}}$ & $11.98^{\mathrm{a}}$ & $1.45^{\mathrm{a}}$ \\
M. oleifera & 100 & $224.43^{\mathrm{ab}}$ & $0.0180^{\mathrm{b}}$ & $3.6325^{\mathrm{ab}}$ & $796.33^{\mathrm{ab}}$ & $11.51^{\mathrm{a}}$ & $1.38^{\mathrm{a}}$ \\
& 200 & $213.31^{\mathrm{b}}$ & $0.0182^{\mathrm{b}}$ & $3.6990^{\mathrm{ab}}$ & $794.52^{\mathrm{ab}}$ & $11.40^{\mathrm{a}}$ & $1.31^{\mathrm{a}}$ \\
& 300 & $198.92^{\mathrm{bc}}$ & $0.0205^{\mathrm{ab}}$ & $3.7732^{\mathrm{a}}$ & $767.05^{\mathrm{bc}}$ & $11.41^{\mathrm{a}}$ & $1.36^{\mathrm{a}}$ \\
& 400 & $184.81^{\mathrm{c}}$ & $0.0227^{\mathrm{a}}$ & $3.6097^{\mathrm{b}}$ & $749.52^{\mathrm{c}}$ & $11.38^{\mathrm{a}}$ & $1.36^{\mathrm{a}}$ \\
EEM & - & 5.9547 & 0.0009 & 0.0367 & 9.0465 & 0.2621 & 0.0428 \\
Valor- $p$ & - & $<0.0001$ & $<0.0001$ & $<0.0001$ & $<0.0001$ & $<0.0001$ & $<0.0001$ \\
Montrol $v s$ & - & 4989.46 & 0.000022 & 0.0045904 & 3610.49 & 1.2915 & 0.0343 \\
Lineal & - & 8480.20 & 0.000072 & 0.0021170 & 9235.56 & 0.6867 & 0.0191 \\
Cuadrático & - & 24.945 & 0.000007 & 0.0065361 & 301.66 & 0.7376 & 0.0191 \\
\hline
\end{tabular}

$b=$ Asíntota de producción de gas ( $\mathrm{ml} \mathrm{g}^{-1}$ de MS); c: tasa de producción de gas (/h); L: fase lag (h); DIVMS: degradabilidad in vitro de la MS (mg/g de MS); EM: energía metabolizable (MJ/kg de MS); AGCC: ácidos grasos de cadena corta (mmol)

Valores en la misma columna con diferente literal son estadísticamente significativos Tukey $(p<0.05)$

$\mathrm{EEM}=$ Error estándar de la media

el nivel de inclusión de $M$. oleifera afecta la degradabilidad, donde la dieta testigo obtuvo el menor valor (603.3 $\left.\mathrm{g} \mathrm{kg}^{-1} \mathrm{MS}\right)$. Asimismo, las dietas con niveles de 100,200 y $300 \mathrm{~g} \mathrm{~kg}^{-1}$ MS, fueron superiores (624.2, 642.1 y 651.7 $\mathrm{g} \mathrm{kg}^{-1} \mathrm{MS}$, respectivamente). En la DMS a las $48 \mathrm{~h}$ no se observó efecto del nivel de inclusión.

Para el caso de la DFDN a la hora 24, se observó una disminución en la degradabilidad conforme aumento el nivel de inclusión de $M$. oleifera, no así para la DFDN a la hora 48 donde no se encontraron diferencias significativas con valores dentro del rango de 541 a $592 \mathrm{~g} \mathrm{~kg}^{-1} \mathrm{MS}$. En el caso de la
DFDA a las $24 \mathrm{~h}$ se observa que el nivel de inclusión de $M$. oleifera más elevado (401.7 $\mathrm{g} \mathrm{kg}^{-1} \mathrm{MS}$ ) afecta la degradabilidad, caso contrario en los niveles de $0,100,200$ y $300 \mathrm{~g}$ $\mathrm{kg}^{-1} \mathrm{MS}$, donde no se encontró efecto, mostrando valores que oscilan entre 525 a 548 $\mathrm{g} \mathrm{kg}^{-1} \mathrm{MS}$.

Para la DFDA a las 48 h se encontró efecto cuadrático, observándose que las dietas con niveles de $0,100,300$ y $400 \mathrm{~g} \mathrm{~kg}^{-1}$ MS no mostraron diferencias estadísticas significativas $\left(649.0,639.0,624.7\right.$ y $567.0 \mathrm{~g} \mathrm{~kg}^{-1}$ MS, respectivamente). La dieta con el nivel de inclusión de $200 \mathrm{~g} \mathrm{~kg}^{-1} \mathrm{MS}$ obtuvo el valor más bajo de degradabilidad (532 $\left.\mathrm{g} \mathrm{kg}^{-1} \mathrm{MS}\right)$. 
Cuadro 4. Niveles de Moringa oleifera en dietas para corderos en crecimiento sobre la degradabilidad in vitro de la materia seca y fibra a las 24 y $48 \mathrm{~h}$ de incubación en las botellas de fermentación

\begin{tabular}{|c|c|c|c|c|c|c|c|}
\hline \multirow[b]{2}{*}{$\begin{array}{l}\text { Dietas } \\
\left(\mathrm{g} \mathrm{kg}^{-1} \mathrm{MS}\right)\end{array}$} & \multirow{2}{*}{$\begin{array}{l}\text { Nivel de } \\
\text { inclusión } \\
\left(\mathrm{g} \mathrm{kg}^{-1}\right. \\
\mathrm{MS})\end{array}$} & \multicolumn{6}{|c|}{ Degradabilidad in vitro $\left(\mathrm{g} \mathrm{kg}^{-1} \mathrm{MS}\right)$} \\
\hline & & $\begin{array}{c}\text { DMS } \\
24 \mathrm{~h}\end{array}$ & $\begin{array}{c}\text { DMS } \\
48 \mathrm{~h}\end{array}$ & $\begin{array}{c}\text { DFDN } \\
24 \mathrm{~h}\end{array}$ & $\begin{array}{c}\text { DFDN } \\
48 \mathrm{~h}\end{array}$ & $\begin{array}{c}\text { DFDA } \\
24 \mathrm{~h}\end{array}$ & $\begin{array}{c}\text { DFDA } \\
48 \mathrm{~h}\end{array}$ \\
\hline Testigo & 0 & $603.3^{c}$ & $771.2^{\mathrm{a}}$ & $393.2^{\mathrm{a}}$ & $592.0^{\mathrm{a}}$ & $548.3^{\mathrm{a}}$ & $649.0^{\mathrm{a}}$ \\
\hline M. oleifera & 100 & $624.2^{\mathrm{abc}}$ & $772.7^{\mathrm{a}}$ & $302.0^{\mathrm{b}}$ & $571.5^{\mathrm{a}}$ & $525.7^{\mathrm{a}}$ & $639.0^{\mathrm{ab}}$ \\
\hline & 200 & $642.1^{\mathrm{ab}}$ & $741.1^{\mathrm{a}}$ & $301.0^{\mathrm{b}}$ & $547.3^{\mathrm{a}}$ & $540.6^{\mathrm{a}}$ & $532.0^{\mathrm{b}}$ \\
\hline & 300 & $651.7^{\mathrm{a}}$ & $763.5^{\mathrm{a}}$ & $283.5^{\mathrm{b}}$ & $541.5^{\mathrm{a}}$ & $532.5^{\mathrm{a}}$ & $624.7^{\mathrm{ab}}$ \\
\hline & 400 & $611.3^{\mathrm{bc}}$ & $760.7^{\mathrm{a}}$ & $313.4^{\mathrm{b}}$ & $555.0^{\mathrm{a}}$ & $401.7^{\mathrm{b}}$ & $567.0^{\mathrm{ab}}$ \\
\hline Error estándar & - & 0.7647 & 0.8480 & 1.5468 & 1.1094 & 1.5571 & 2.4414 \\
\hline Valor- $p$ & - & 0.0057 & 0.1386 & 0.0047 & 0.1283 & 0.0003 & 0.0282 \\
\hline $\begin{array}{l}\text { Testigo vs } \\
\text { M. oleifera }\end{array}$ & - & 0.0068 & 0.2470 & 0.0004 & 0.0470 & 0.0200 & 0.0585 \\
\hline Lineal & - & 0.1018 & 0.2879 & 0.0045 & 0.0380 & 0.0002 & 0.0437 \\
\hline Cuadrático & - & 0.0010 & 0.1827 & 0.0049 & 0.1411 & 0.0021 & 0.2804 \\
\hline
\end{tabular}

DMS = degradabilidad in vitro de la materia seca; DFDN = degradabilidad in vitro de la fibra detergente neutro; DFDA = degradabilidad in vitro de la fibra detergente acido $\mathrm{EEM}=$ Error estándar de la media Valores en la misma columna con diferente literal son estadísticamente significativos Tukey $(p<0.05)$

\section{Discusión}

\section{Producción de Gas in vitro}

Con relación a la producción de gas, se pudo observar que a medida que se incrementó la inclusión de $M$. oleifera disminuyó la producción de gas a las 48 y $72 \mathrm{~h}$ de incubación. Resultados contrarios fueron mencionados por Foidl et al. (2001) quienes observaron que al incrementar los niveles de soya a diferentes se incrementaba la producción de gas, y que este aumento no era un indicio de un mejor aprovechamiento del alimento.
Estos resultados concuerdan con los reportados por Molina-Botero et al. (2013), quienes mencionaron que a las $48 \mathrm{~h}$ no observaron diferencias en acumulación de gas entre las muestras $(\mathrm{p}=0.86)$, donde todos los forrajes y sus mezclas alcanzaron producciones de alrededor de $115 \mathrm{ml}$ de gas $\mathrm{g}^{-1} \mathrm{MO}$, lo cual corresponde al $77-83 \%$ del gas total producido durante el experimento; así mismo, mencionan que a medida que el proceso fermentativo avanza, el material es hidratado y colonizado por los microorganismos ruminales lo que origina diferentes tasas de degradación, dependiendo de la concentración de carbohidratos estructurales. 
Por su parte, Montejo et al. (2012) en un estudio realizado con soya difieren con los resultados obtenidos en el presente estudio, reportando que a medida que aumentó el porcentaje de inclusión de soya se incrementó la producción de gas in vitro, posiblemente debido a las características de los alimentos incubados. Estos autores indican que dicho comportamiento es debido a que, al incubar una mayor cantidad de materia seca, hay más materia prima para el desarrollo de la actividad microbiana y ello trae consigo un aumento de la degradación del alimento, lo cual se percibe en un incremento de la producción de gas.

Por otro lado, Melesse (2001) reporta valores más altos de producción de gas in vitro (47.9 ml) para $M$. stenopetala que para M. oleifera $(40.6 \mathrm{ml})$. Asimismo, obtiene valores más altos de EM, DMO y de ácidos grasos de cadena corta para $M$. stenopetala que para $M$. oleifera.

\section{Digestibilidad in vitro}

Los resultados de DIVMS no fueron constantes y más bien diferentes a los obtenidos por Molina-Botero et al. (2013) con gramíneas y leguminosas, quienes observaron un incremento de la DIVMS con el aumento de la inclusión de leguminosas, especialmente en animales en confinamiento, probablemente debido al bajo contenido de proteína en el pasto ángleton evaluado en ese estudio.

Similar situación fue reportada por García et al. (2006) trabajando con moringa, morera y leucaena, al obtener mayor degradación de la MS y la MO para moringa. Generalmente, las diferencias en la degradabilidad se relacionaron con sus contenidos de fibra y PB, debido a que estas especies tienen poca presencia de metabolitos tóxicos y bajas concentraciones de posibles factores anti-nutricionales (Rodríguez et al., 2014).

Por último, Gutiérrez et al. (2015) observaron que la concentración de PB fue de $19.5 \%$ y menor de FDN (56.0\%) en $M$. olifeira. En el presente estudio, los parámetros $(\mathrm{a}, \mathrm{b}, \mathrm{a}+\mathrm{b})$ generados por el modelo matemático de degradación indicaron efectos significativos, donde el $60 \%$ de moringa en la mezcla mixta alcanza el valor más elevado de degradación de la MS con 69.8\%.

\section{Conclusiones}

Los resultados de producción de gas y degradabilidad in vitro sugieren utilizar niveles de inclusión de M. oleifera por debajo del $30 \%$ en dietas para corderos en crecimiento.

\section{Agradecimientos}

Los autores agradecen al Consejo Nacional de Ciencia y Tecnología (CONACyT) por la beca otorgada al primer autor para realizar estudios de doctorado.

\section{Literatura Citada}

1. Astutia DA, Babab AS, Wibawanc IWT. 2011. Rumen fermentation, blood metabolites, and performance of sheep fed tropical browse plants. J Anim Sci Technol 34: 201-206. doi: 10.5398/ medpet.2011.34.3.201

2. Debela E, Tolera A. 2013. Nutritive value of botanical fractions of Moringa oleifera and Moringa stenopetala grown in the mid-rift valley of southern Ethiopia. Agroforesty Systems 87:11471155. doi: 10.1007/s10457-013-9626-9

3. Ceballos A, Noguera R, Bolívar DM, Posada SL. 2008. Comparación de las técnicas in situ de los sacos de nylon e in vitro (Daisy ${ }^{\text {II) }}$ para estimar la cinética de degradación de alimentos para rumiantes. Livestock Res Rural Develop 20(7). [Internet]. Disponible: http:// www.lrrd.org/lrrd20/7/ceba20108.htm

4. Foidl N, Makkar HPS, Becker K. 2001. The potential of Moringa oleifera for agricultural and industrial 
uses. In: Fuglie LJ (ed). The miracle tree. The multiple attributes of moringa. Dakar, Senegal. [Internet]. Available in: https:// miracletrees.org/moringa-doc/the_potential_of_moringa_oleifera_for_agricultural_and_industrial_uses.pdf

5. France J, Dijksträ J, Dhañoa MS, Lopez S, Bannink A. 2000. Estimating the extent of degradation of ruminant feeds from a description of their gas production profiles observed in vitro: derivation of models and other mathematical considerations. Br J Nutr 83: 143-150. doi: 10.1017/s0007114500000180

6. García DE, Medina MG, Humbría J, Domínguez C, Baldizán A, Cova L, Soca M. 2006. Composición proximal, niveles de metabolitos secundarios y valor nutritivo del follaje de algunos árboles forrajeros tropicales. Arch Zootec 55: 373-384.

7. Gutiérrez D, Borjas-Rojas E, Rodríguez-Hernández R, Rodríguez $Z$, Stuart R, Sarduy L. 2015. Evaluación de la composición química y degradabilidad ruminal in situ de ensilaje mixto con Pennisetum purpureum cv Cuba CT-169: Moringa oleifera. Av Investig Agropecu 19: 7-16.

8. [INEGI] Instituto Nacional de Estadística y Geografía. 2019. https:// www.inegi.org.mx/temas/climatologia/.

9. Melesse A. 2001. Comparative assessment on chemical compositions and feeding values of leaves of Moringa stenopetala and Moringa oleifera using in vitro gas production method. Eth J Appl Sci Technol 2: 31-41.

10. Menke KH, Steingass H. 1988. Estimation of the energetic feed value obtained from chemical analysis and in vitro gas production using rumen fluid. Anim Res Dev 28: 7-55.

11. Molina-Botero IC, Cantet JM, Montoya S, Correa-Londoño GA, Barahona-Rosales R. 2013. Producción de metano in vitro de dos gramíneas tropicales solas y mezcladas con Leucaena leucocephala o Gliricidia sepium. Rev CES Med Vet Zootec 8: 15-31.

12. Montejo IL, López O, Sánchez T, Muetzel S, Becker K, Lamela L. 2012. Effect of the soybean inclusion rate on the in vitro digestibility of the meal from Moringa oleifera pods. Pastos y Forrajes 35: 197-204.

13. Pell AN, Doane PH, Schofield P.1997. In vitro digestibility and gas production. In: Simpósio sobre Tópicos Especiais em Zootecnia. Lavras, Brazil.

14. Posada SL, Noguera RR. 2005. Técnica in vitro de producción de gases: una herramienta para la evaluación de alimentos para rumiantes. Livestock Res Rural Dev 17(4). [Internet]. Disponible en: http://www.lrrd.org//rrd17/4/posa17036.htm

15. Quintanilla-Medina J, JoaquínCancino S, Martínez-González, J, Limas-Martínez A, López-Aguirre D, Estrada-Drouaillet B, et al. 2018. Usos de Moringa oleifera Lam. (Moringaceae) en la alimentación de rumiantes. Agroproductividad 11: 89-93.

16. Rodríguez PR. 2011. Alimentación de vacas lecheras con Moringa oleifera fresco o ensilado y su efecto sobre la producción, composición y calidad de leche. Tesis de Maestría. Nicaragua: Univ. Nacional Agraria. Nicaragua. $35 \mathrm{p}$.

17. Rodríguez R, González N, Alonso J, Domínguez M, Sarduy L. 2014. Valor nutritivo de harinas de follaje de cuatro especies arbóreas tropicales para rumiantes. Rev Cubana Cienc Agr 48: 371-377.

18. SAS Institute. 2002. SAS User's guide: ststistics v. 9.0. Institute. Cary NC, USA. $956 \mathrm{p}$.

19. Theodorou MK, Williams BA, Dhanoa MS, McAllan AB, France J. 1994. A simple gas production method using a pressure transducer to determine the fermentation kinetics of ruminant feeds. Anim Feed Sci Technol 48: 185-197. doi: 10.1016/0377-8401(94)90171-6 